\title{
Sequence Variation in HSP40 Gene among 16 Toxoplasma gondii Isolates from Different Hosts and Geographical Locations
}

\author{
Zhong-Yuan Li, ${ }^{1,2}$ Jing Lu, ${ }^{1,3}$ Dong-Hui Zhou, ${ }^{1}$ Jia Chen, ${ }^{1}$ and Xing-Quan Zhu ${ }^{1,2}$ \\ ${ }^{1}$ State Key Laboratory of Veterinary Etiological Biology, Key Laboratory of Veterinary Parasitology of Gansu Province, \\ Lanzhou Veterinary Research Institute, Chinese Academy of Agricultural Sciences, Lanzhou, Gansu 730046, China \\ ${ }^{2}$ College of Animal Science and Veterinary Medicine, Heilongjiang Bayi Agricultural University, Daqing, Heilongjiang 163319, China \\ ${ }^{3}$ Guangdong Dahuanong Animal Health Products Co., Ltd., Yunfu, Guangdong 527400, China
}

Correspondence should be addressed to Xing-Quan Zhu; zhuxingquan@caas.cn

Received 10 July 2015; Accepted 19 October 2015

Academic Editor: Somboon Tanasupawat

Copyright (c) 2015 Zhong-Yuan Li et al. This is an open access article distributed under the Creative Commons Attribution License, which permits unrestricted use, distribution, and reproduction in any medium, provided the original work is properly cited.

Toxoplasma gondii with worldwide distribution has received substantial medical and scientific attentions as it causes serious clinical and veterinary problems especially for pregnant women and immunocompromised patients. Heat shock protein 40 (HSP40) plays a variety of essential roles in the pathogenesis of this protozoan parasite. In order to detail the genetic diversity of HSP40 gene, 16 T. gondii strains from different hosts and geographical locations were used in this study. Our results showed that HSP40 sequence of the examined strains was between $6621 \mathrm{bp}$ and $6644 \mathrm{bp}$ in length, and their A+T content was from $48.54 \%$ to $48.80 \%$. Furthermore, sequence analysis presented 195 nucleotide mutation positions (0.12\%-1.14\%) including 29 positions in CDS (0.02\%0.12\%) compared with T. gondii ME49 strain (ToxoDB: TGME49_265310). Phylogenetic assay revealed that T. gondii strains representing three classical genotypes (Types I, II, and III) were completely separated into different clusters by maximum parsimony (MP) method, but Type II and ToxoDB\#9 strains were grouped into the same cluster. These results suggested that HSP40 gene is not a suitable marker for T. gondii population genetic research, though three classical genotypes of T. gondii could be differentiated by restriction enzymes $M s c \mathrm{I}$ and EarI existing in amplicon C.

\section{Introduction}

Toxoplasma gondii infects almost all the warm-blooded animals including about one-third population of humans $[1,2]$ and can cause serious clinical diseases, especially in pregnant women and immunocompromised individuals such as tumor sufferers and AIDS patients $[3,4]$. T. gondii can also cause abortion and congenital toxoplasmosis in livestock, leading to considerable economic losses $[5,6]$.

Heat shock proteins (HSPs) involved in antigen presentation and cross-presentation play important roles in activation of immune-related cells such as macrophages, lymphocytes, and DCs [7-9]. As the important member of HSPs, HSP40 associated with DNA replication, protein folding, assembling and degradation, translocation across membranes, signal transduction, and endocytosis participates in the pathogenesis of apicomplexan parasites such as Plasmodium falciparum [10, 11]. Recent studies emphasized that different clonal types of T. gondii strains with diverse geographical distribution can cause different toxoplasmosis in animals and humans $[12,13]$. In order to unveil the details of $T$. gondii genetic diversity, sequence variation of the type II HSP40TgSIS1 (ToxoDB: TGME49_265310, previously named TGME49_065310) [14] among T. gondii isolates from different hosts and geographical regions was examined in this study.

\section{Materials and Methods}

2.1. T. gondii Isolates and gDNA Preparation. Sixteen T. gondii isolates harvested from different hosts and geographical locations were used in the present study (Table 1). Genomic DNA was extracted as normal and stored at $-20^{\circ} \mathrm{C}$ till used.

2.2. PCR Amplification and Sequencing. Three fragments (A, B, and C) (Figure 1) were separated based on the 
TABLE 1: Details of Toxoplasma gondii isolates used in this study.

\begin{tabular}{lcccc}
\hline Number & Isolate & Host & Geographical location & Genotype $^{*}$ \\
\hline 1 & CTG & Cat & United States & Reference, Type III, ToxoDB\#2 \\
2 & TgCatBr64 & Cat & Brazil & Reference, ToxoDB\#111 \\
3 & GT1 & Goat & United States & Reference, Type I, ToxoDB\#10 \\
4 & Human & Pig & France & Reference, Type I, ToxoDB\#10 \\
5 & TgPLH & Toucan & Henan, China & Type I, ToxoDB\#10 \\
6 & TgToucan & Human & Costa Rica & Reference, ToxoDB\#52 \\
7 & MAS & Cat & France & Reference, ToxoDB\#17 \\
8 & TgCatBr5 & Sheep & Brazil & Reference, ToxoDB\#19 \\
9 & PTG & Human & United States & Reference, Type II, ToxoDB\#1 \\
10 & PRU & Sheep & France & Type II, ToxoDB\#1 \\
11 & QHO & Cat & Guangzhou, China & Type II, ToxoDB\#1 \\
12 & TgC7 & Pig & Panyu, China & ToxoDB\#9 \\
13 & PYS & Pig & Jingyuan, Gansu, China & ToxoDB\#9 \\
14 & GJS & Cougar & Canada & ToxoDB\#9 \\
15 & TgCgCal & USA & Reference, ToxoDB\#66 \\
16 & TgWtdSc40 & Type 12, ToxoDB\#5 \\
\hline
\end{tabular}

${ }^{*}$ Based on the results of Zhou et al. [25, 26] and Su et al. [20].

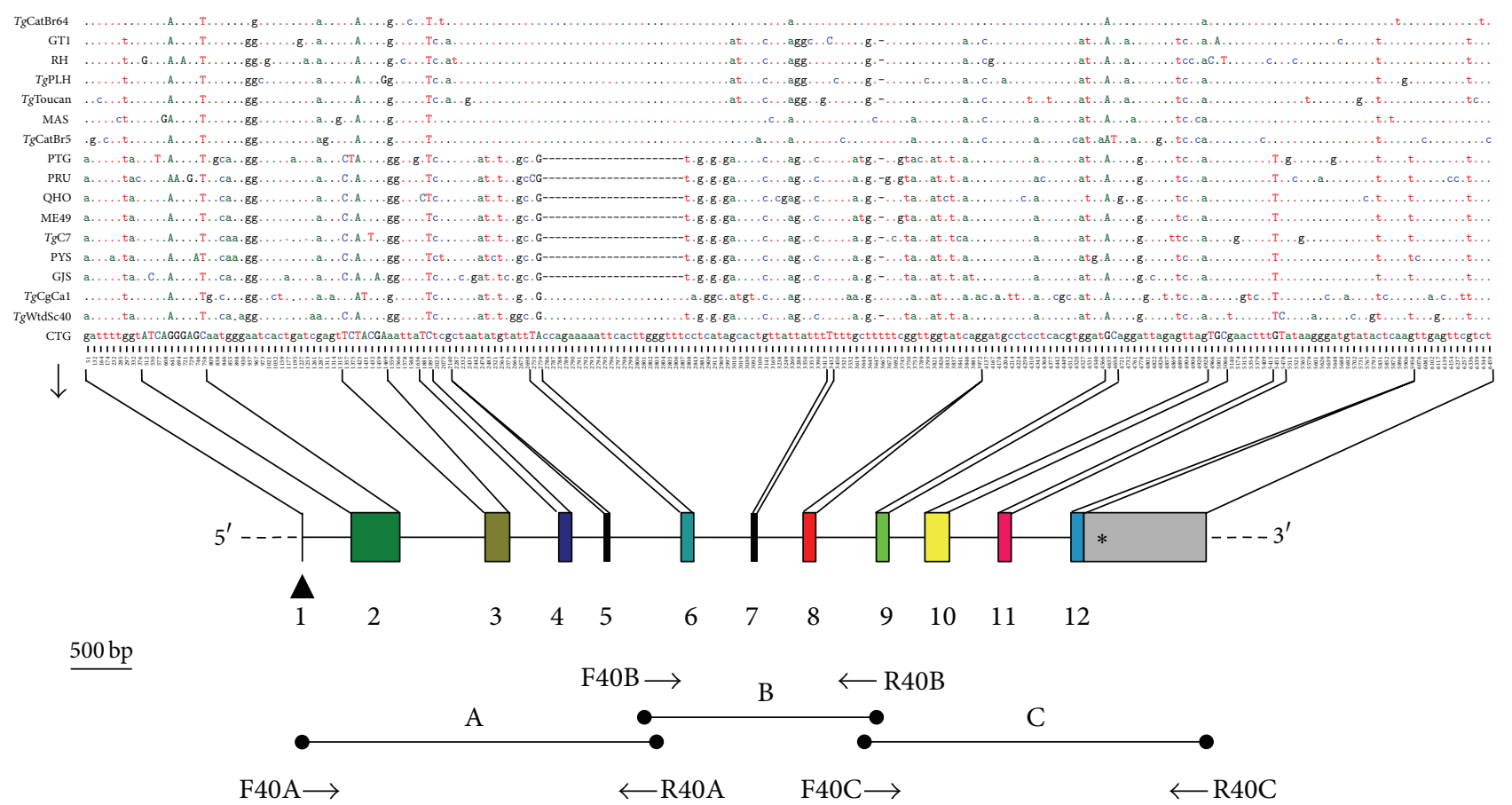

FIGURE 1: Sequence analysis of HSP40 gene among different Toxoplasma gondii strains. Color boxes indicate 12 extrons of HSP40 gene and the gray one stands for the region of $3^{\prime}$-UTR. Black triangle $(\mathbf{\Delta})$ and asterisk $(*)$ indicate the translation start condon (ATG) and the stop one (TAG), respectively. Three pairs of specific primers (F40A/R40A, F40B/R40B, and F40C/R40C) used in this study were marked with arrows. Upper or lower case, respectively, indicates different nucleotide of extron or intron $/ 3^{\prime}$-UTR. Point (.) and stub (-) among different nucleotides indicate identical or no nucleotide here compared with that of CTG strain (bottom line). The number beside the sequence stands for the variable sequence position for nucleotide, and black bar indicates $500 \mathrm{bp}$ in length.

HSP40 sequence of T. gondii ME49 isolate (ToxoDB: TGME49_265310), and the amplifications were performed by PCR using three pairs of specific primers, respectively (Table 2). Thermal cycling conditions were according to the following protocol: initial denaturation at $94^{\circ} \mathrm{C}$ for $10 \mathrm{~min}$ followed by 35 cycles composing of $94^{\circ} \mathrm{C}$ for $1 \mathrm{~min}, 54.7^{\circ} \mathrm{C}$ (amplicon A), $66.8^{\circ} \mathrm{C}$ (amplicon B) or $64.0^{\circ} \mathrm{C}$ (amplicon $\mathrm{C}$ ) for $45 \mathrm{~s}$ respectively, and $72^{\circ} \mathrm{C}$ for $2 \mathrm{~min}$, and the additional extension step was carried out at $72^{\circ} \mathrm{C}$ for $10 \mathrm{~min}$. Negative control without gDNA was included in each amplification. 
TABLE 2: Details of primers used in this study.

\begin{tabular}{ll}
\hline Primer & Sequence $\left(5^{\prime}-3^{\prime}\right)$ \\
\hline F40A & ATGGGGAAGGTAATCACATT \\
R40A & CGACTGGAACTATCGATTTC \\
F40B & TGTGGGGCGAGAGCCAGAGG \\
R40B & TGCAGAGGTGCCTTGCGTTT \\
F40C & CCCTGCATCGCGTGAGCTTC \\
R40C & AACGAGTGGAAAGCCCCCGT \\
\hline
\end{tabular}

Amplicons A, B, and $\mathrm{C}$ were amplified from different $T$. gondii strains by PCR using F40A/R40A, F40B/R40B, and F40C/R40C, respectively.

PCR amplifications were confirmed by agarose gel electrophoresis as previously described [15]. All the PCR products were purified using spin columns (Promega, Madison, USA), ligated with pMD18-T vector (TaKaRa, Dalian, China), and transformed into E. coli DH5 $\alpha$ competent cells (Promega) according to the manufacturers' instructions. The positive colonies confirmed by PCR were sequenced by Shanghai Sangon Biological Engineering Biotechnology Company. All the experiments were run in triplicate.

2.3. Sequence Analysis and Phylogenetic Reconstruction. The sequences of all the examined T. gondii strains were amplified step by step and sequenced. Alignment analysis based on the obtained sequences including the reference one (ToxoDB: TGME49_265310) was carried out with Clustal X 1.83 [16], and the number of sequence variation compared with ME49 strain was calculated as previously described [17]. Phylogenetic reconstructions were performed by maximum parsimony (MP) method using PAUP* 4.0b10 [18], and 100 random addition searches using tree-bisection reconnection (TBR) were carried out for each MP assay. Bootstrap probability (BP) was calculated from 1,000 bootstrap replicates with 10 random additions per replicate in PAUP.

2.4. Characterization of T. gondii Isolates by PCR-RFLP. To evaluate whether HSP40 gene was suitable for genotyping of T. gondii isolates, PCR-RFLP method was also used in this study as previously described $[19,20]$. All the PCR products of amplicon $\mathrm{C}$ were digested with two restriction enzymes $M s c \mathrm{I}$ and EarI by incubating at $37^{\circ} \mathrm{C}$ for $4 \mathrm{~h}$ according to the manufacturer's instructions (NEB, Beijing, China). And the restriction fragments were separated by electrophoresis as previously described [21].

\section{Results and Discussion}

Our results showed that HSP40 gene of all the examined strains was between $6621 \mathrm{bp}$ and $6644 \mathrm{bp}$ in length spliced by amplicons A (2430 bp), B (2107-2130 bp), and C (2252 bp), and their $\mathrm{A}+\mathrm{T}$ contents varied from $48.54 \%$ to $48.80 \%$ (Figure 1, Table 3). The alignment of all the 17 sequences revealed nucleotide mutations at 195 positions $(0.12 \%-1.14 \%)$ in HSP 40 genomic locations and 29 positions in CDS (0.02\%$0.12 \%$ ) in comparison with T. gondii ME49 strain (ToxoDB: TGME49_265310), which was lower than our previous reports of GRA5 [21], ROP38 [22], ROP47 [23], eIF4A [15], and other genes of T. gondii, such as GRA6 [24]. Moreover, 141 transitions $(\mathrm{A} \leftrightarrow \mathrm{G}$ and $\mathrm{C} \leftrightarrow \mathrm{T})$ and 54 transversions $(\mathrm{A} \leftrightarrow$ $\mathrm{C}, \mathrm{A} \leftrightarrow \mathrm{T}, \mathrm{G} \leftrightarrow \mathrm{T}$, and $\mathrm{G} \leftrightarrow \mathrm{C})(R=$ transition/transversion $=2.6$ ) were also identified, and the distance of evolutionary divergence was $0.1 \%-1.0 \%$ among the examined $T$. gondii strains (Table 3).

Nucleotide polymorphisms analysis revealed two polymorphic restriction sites $\mathrm{MscI}$ and EarI in the sequence of amplicon C (2252 bp in length), which can differentiate three classical genotypes of T. gondii (Types I, II, and III) (Figure 2) $[19,20,25,26]$. In brief, the PCR products of $\mathrm{Tg}$ Toucan, MAS, TgCatBr5, and T. gondii Type I strains (GT1, RH, and TgPLH) were digested into four segments (81, 165, 811, and $1195 \mathrm{bp})$; Type II strains (PTG, PRU, and QHO), ToxoDB\#9 (TgC7, PYS, and GJS), TgCgCa1, and TgWtdSc40 were composed of three parts $(81,811$, and $1360 \mathrm{bp})$; and the PCR products of Type III (CTG) and TgCatBr64 were cut into five sections $(81,165,381,811$, and $814 \mathrm{bp})$. The results suggested that all the examined T. gondii strains could not be completely separated into their own groups by PCR-RFLP especially for ToxoDB\#9 strains.

Phylogenetic reconstruction was constructed based on HSP40 sequences of the $17 \mathrm{~T}$. gondii strains including $T$. gondii ME49 isolates (ToxoDB: TGME49_265310) (Figure 3) [18]. Our results showed that T. gondii strains belonging to Type II (PRU, QHO, ME49, and PTG), Type 12 (TgWtdSc40), or ToxoDB\#9 (PYS, TgC7 and GJS) were grouped into the same cluster, whereas TgToucan (ToxoDB\#52) was gathered into the cluster of Type I (RH, GT1, and TgPLH), suggesting that the examined T. gondii strains could not be completely separated by MP method though three classical genotypes of T. gondii (Types I, II, and III) were clustered into different groups.

\section{Conclusion}

Our data suggested that HSP40 gene is not a suitable marker for $T$. gondii population genetic study, though three classical genotypes of T. gondii (Types I, II, and III) could be differentiated by polymorphic restriction endonuclease sites MscI and EarI existing in amplicon C.

\section{Conflict of Interests}

All the authors declare no conflict of interests.

\section{Authors' Contribution}

Zhong-Yuan Li and Jing Lu contributed equally to this work.

\section{Acknowledgments}

This project support was provided, in part, by the National Natural Science Foundation of China (Grant no. 31172316) 


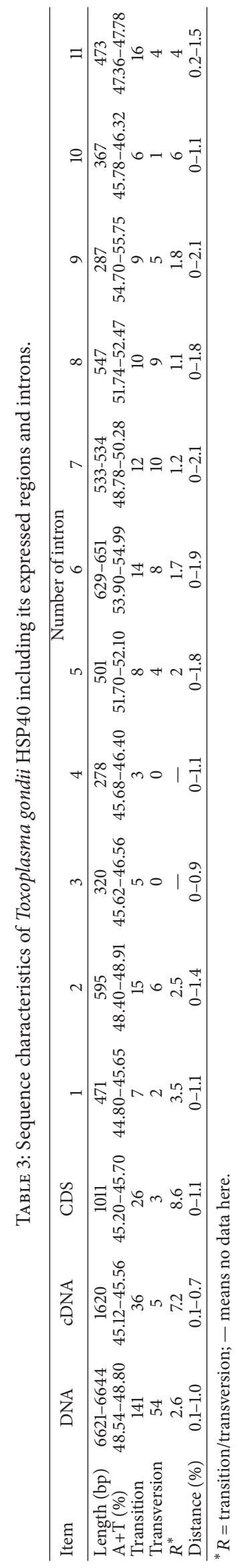




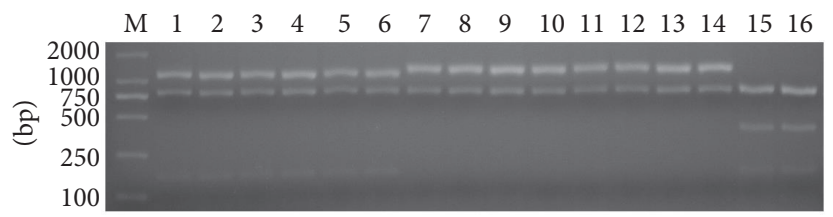

Figure 2: PCR-RFLP analysis based on the PCR products of amplicon C. Lane M indicates DL2000; lanes 1-16 stand for T. gondii Type I (GT1, RH, and TgPLH); TgToucan, MAS, and TgCatBr5, Type II (PTG, PRU, and QHO); ToxoDB\#9 (TgC7, PYS, and GJS), TgCgCal, and $\mathrm{TgWtdSc40}$, Type III (CTG); and $\mathrm{TgCatBr64}$, respectively.

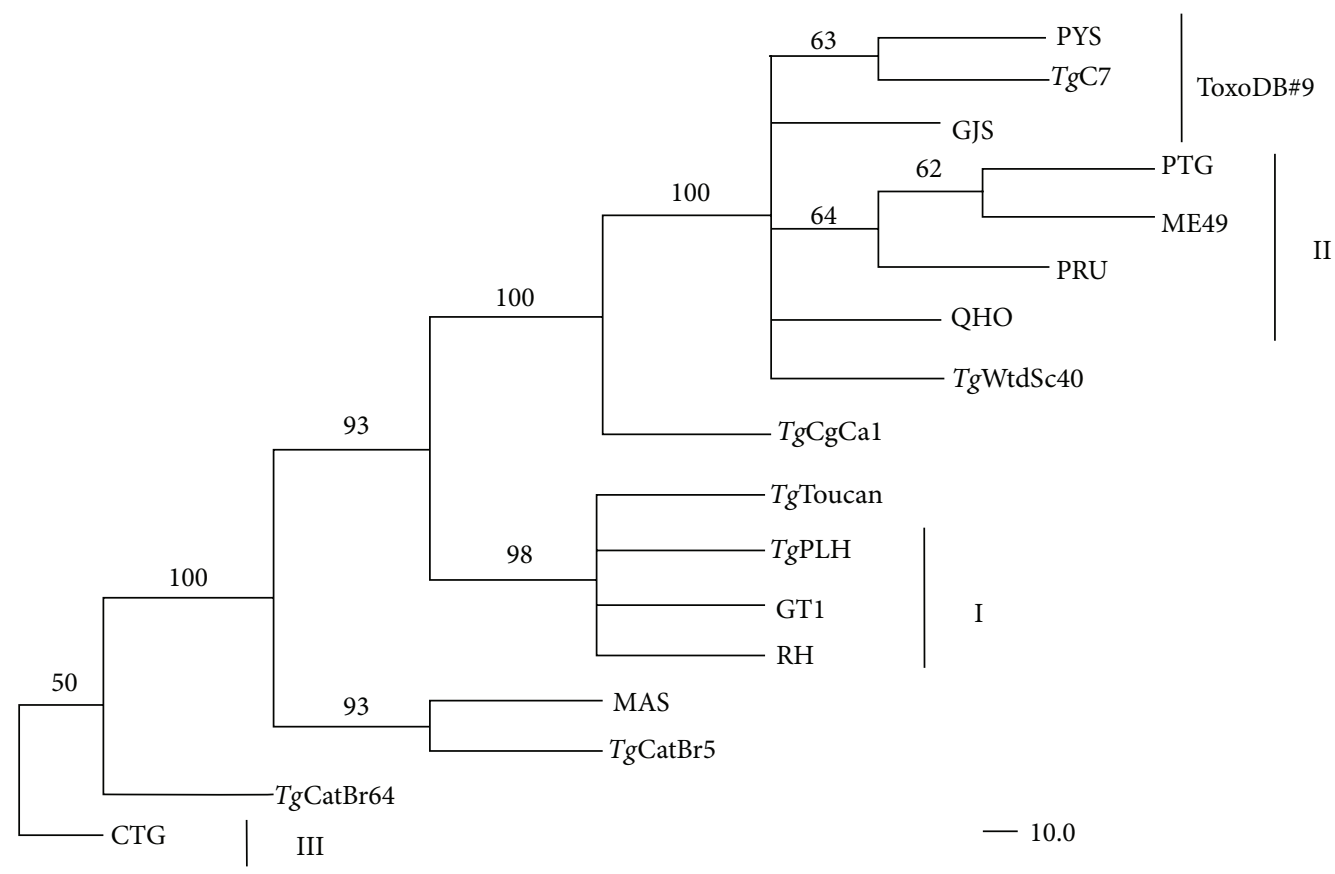

FIGURE 3: Phylogenetic analysis of HSP40 gene among 17 Toxoplasma gondii strains using maximum parsimony (MP) method. Three clusters of the classical genotypes (Types I, II, and III) and ToxoDB\#9 were denoted. The numbers along branches indicate bootstrap values (\%).

and the Science Fund for Creative Research Groups of Gansu Province (Grant no. 1210RJIA006).

\section{References}

[1] J. G. Montoya and O. Liesenfeld, “Toxoplasmosis," The Lancet, vol. 363, no. 9425, pp. 1965-1976, 2004.

[2] D. Schlüter, W. Däubener, G. Schares, U. Groß, U. Pleyer, and C. Lüder, "Animals are key to human toxoplasmosis," International Journal of Medical Microbiology, vol. 304, no. 7, pp. 917-929, 2014.

[3] K. Kim and L. M. Weiss, "Toxoplasma: the next 100 years," Microbes and Infection, vol. 10, no. 9, pp. 978-984, 2008.

[4] L. M. Weiss and J. P. Dubey, "Toxoplasmosis: a history of clinical observations," International Journal for Parasitology, vol. 39, no. 8, pp. 895-901, 2009.

[5] J. P. Dubey, Toxoplasmosis of Animals and Humans, CRC Press, Boca Raton, Fla, USA, 2nd edition, 2010.

[6] P. Zhou, Z. Chen, H.-L. Li et al., "Toxoplasma gondii infection in humans in China," Parasites \& Vectors, vol. 4, article 165, 2011.
[7] Z. Li, A. Menoret, and P. Srivastava, "Roles of heat-shock proteins in antigen presentation and cross-presentation," Current Opinion in Immunology, vol. 14, no. 1, pp. 45-51, 2002.

[8] G. Kaul and H. Thippeswamy, "Role of heat shock proteins in diseases and their therapeutic potential," Indian Journal of Microbiology, vol. 51, no. 2, pp. 124-131, 2011.

[9] T. J. Borges, L. Wieten, M. J. C. van Herwijnen et al., "The antiinflammatory mechanisms of Hsp70," Frontiers in Immunology, vol. 3, article 95, 2012.

[10] P. Acharya, S. Chaubey, M. Grover, and U. Tatu, "An exported heat shock protein 40 associates with pathogenesis-related knobs in Plasmodium falciparum infected erythrocytes," PLoS ONE, vol. 7, no. 9, Article ID e44605, 2012.

[11] S. Külzer, S. Charnaud, T. Dagan et al., "Plasmodium falciparum-encoded exported hsp70/hsp40 chaperone/cochaperone complexes within the host erythrocyte," Cellular Microbiology, vol. 14, no. 11, pp. 1784-1795, 2012.

[12] L. D. Sibley and J. W. Ajioka, "Population structure of Toxoplasma gondii: clonal expansion driven by infrequent recombination and selective sweeps," Annual Review of Microbiology, vol. 62 , pp. 329-351, 2008. 
[13] F. Robert-Gangneux and M.-L. Dardé, "Epidemiology of and diagnostic strategies for toxoplasmosis," Clinical Microbiology Reviews, vol. 25, no. 2, pp. 264-296, 2012.

[14] M. J. Figueras, O. A. Martin, P. C. Echeverria et al., “Toxoplasma gondii Sisl-like J-domain protein is a cytosolic chaperone associated to HSP90/HSP70 complex," International Journal of Biological Macromolecules, vol. 50, no. 3, pp. 725-733, 2012.

[15] J. Chen, S.-F. Fang, D.-H. Zhou, Z.-Y. Li, G.-H. Liu, and X.-Q. $\mathrm{Zhu}$, "Sequence variation in the Toxoplasma gondii eIF4A gene among strains from different hosts and geographical locations," Genetics and Molecular Research, vol. 13, no. 2, pp. 3356-3361, 2014.

[16] J. D. Thompson, T. J. Gibson, F. Plewniak, F. Jeanmougin, and D. G. Higgins, "The CLUSTAL_X windows interface: flexible strategies for multiple sequence alignment aided by quality analysis tools," Nucleic Acids Research, vol. 25, no. 24, pp. 48764882, 1997.

[17] N. B. Chilton, R. B. Gasser, and I. Beveridge, "Differences in a ribosomal DNA sequence of morphologically indistinguishable species within the Hypodontus macropi complex (Nematoda: Strongyloidea)," International Journal for Parasitology, vol. 25, no. 5, pp. 647-651, 1995.

[18] D. L. Swofford, PAUP* . Phylogenetic Analysis Using Parsimony. Version 4.0610, Sinauer Associates, Sunderland, Mass, USA, 2002.

[19] C. Su, X. Zhang, and J. P. Dubey, "Genotyping of Toxoplasma gondii by multilocus PCR-RFLP markers: a high resolution and simple method for identification of parasites," International Journal for Parasitology, vol. 36, no. 7, pp. 841-848, 2006.

[20] C. Su, E. K. Shwab, P. Zhou, X.-Q. Zhu, and J. P. Dubey, "Moving towards an integrated approach to molecular detection and identification of Toxoplasma gondii," Parasitology, vol. 137, no. 1, pp. 1-11, 2010.

[21] J. Chen, Z.-Y. Li, D.-H. Zhou, G.-H. Liu, and X.-Q. Zhu, "Genetic diversity among Toxoplasma gondii strains from different hosts and geographical regions revealed by sequence analysis of GRA5 gene," Parasites \& Vectors, vol. 5, article 279, 2012.

[22] Y. Xu, N. Z. Zhang, J. Chen et al., “Toxoplasma gondii rhoptry protein 38 gene: sequence variation among isolates from different hosts and geographical locations," Genetics and Molecular Research, vol. 13, no. 3, pp. 4839-4844, 2014.

[23] J. L. Wang, T. T. Li, Z. Y. Li, S. Y. Huang, H. R. Ning, and X. Q. Zhu, "Rhoptry protein 47 gene sequence: a potential novel genetic marker for population genetic studies of Toxoplasma gondii," Experimental Parasitology, vol. 154, pp. 1-4, 2015.

[24] A. Fazaeli, P. E. Carter, M. L. Darde, and T. H. Pennington, "Molecular typing of Toxoplasma gondii strains by GRA6 gene sequence analysis," International Journal for Parasitology, vol. 30, no. 5, pp. 637-642, 2000.

[25] P. Zhou, H. Zhang, R.-Q. Lin et al., "Genetic characterization of Toxoplasma gondii isolates from China," Parasitology International, vol. 58, no. 2, pp. 193-195, 2009.

[26] P. Zhou, H. Nie, L.-X. Zhang et al., "Genetic characterization of Toxoplasma gondii isolates from pigs in China," Journal of Parasitology, vol. 96, no. 5, pp. 1027-1029, 2010. 

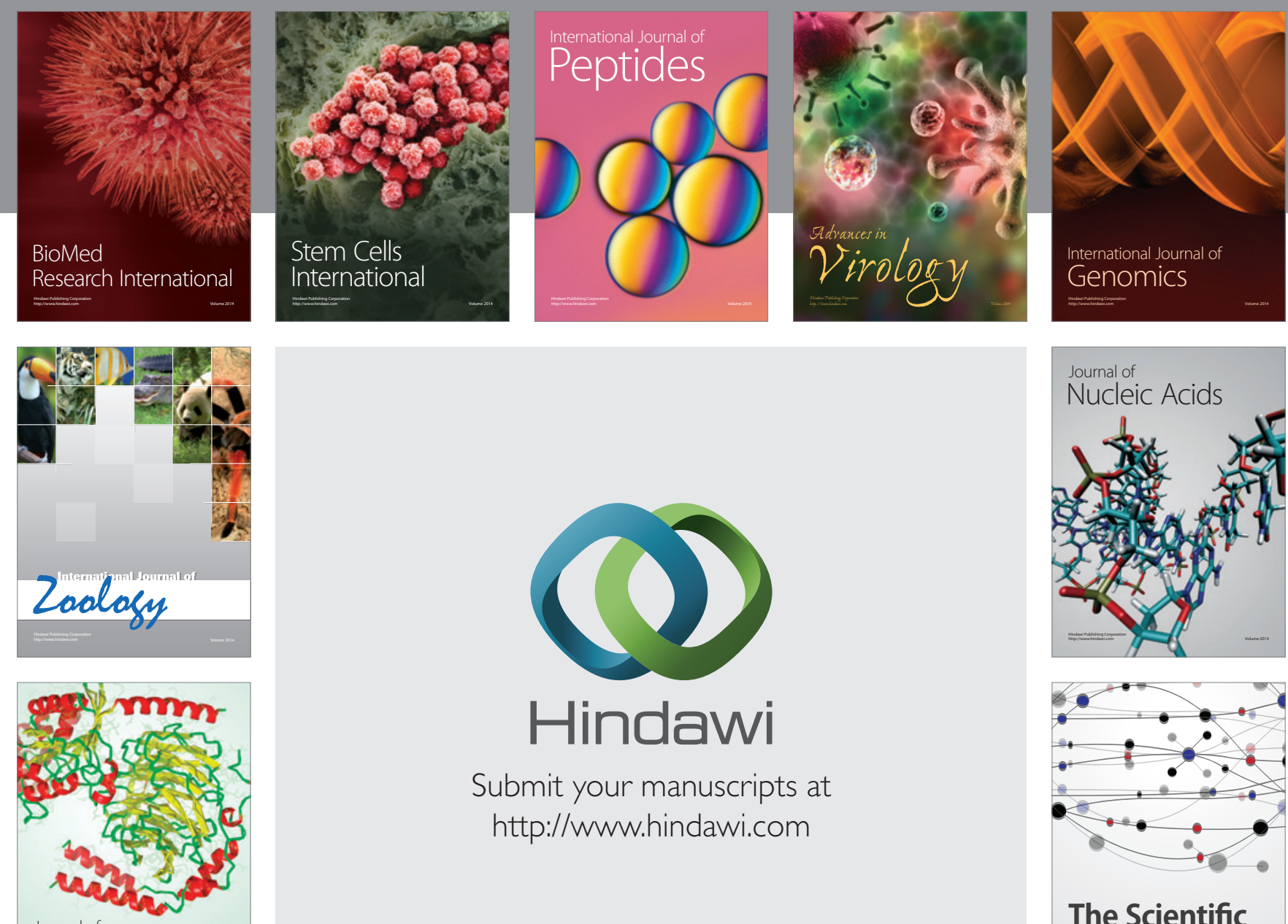

Submit your manuscripts at

http://www.hindawi.com

Journal of
Signal Transduction
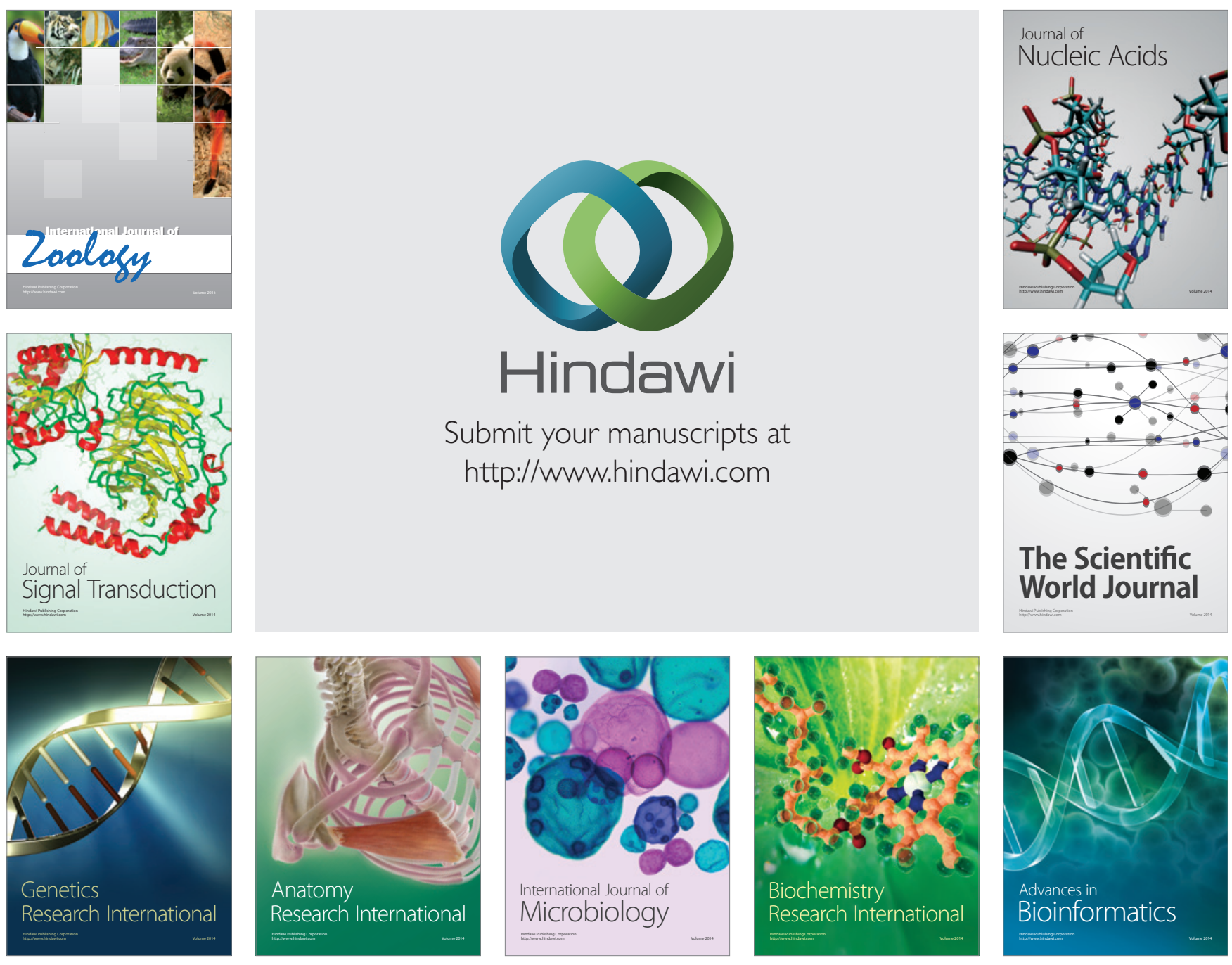

The Scientific World Journal
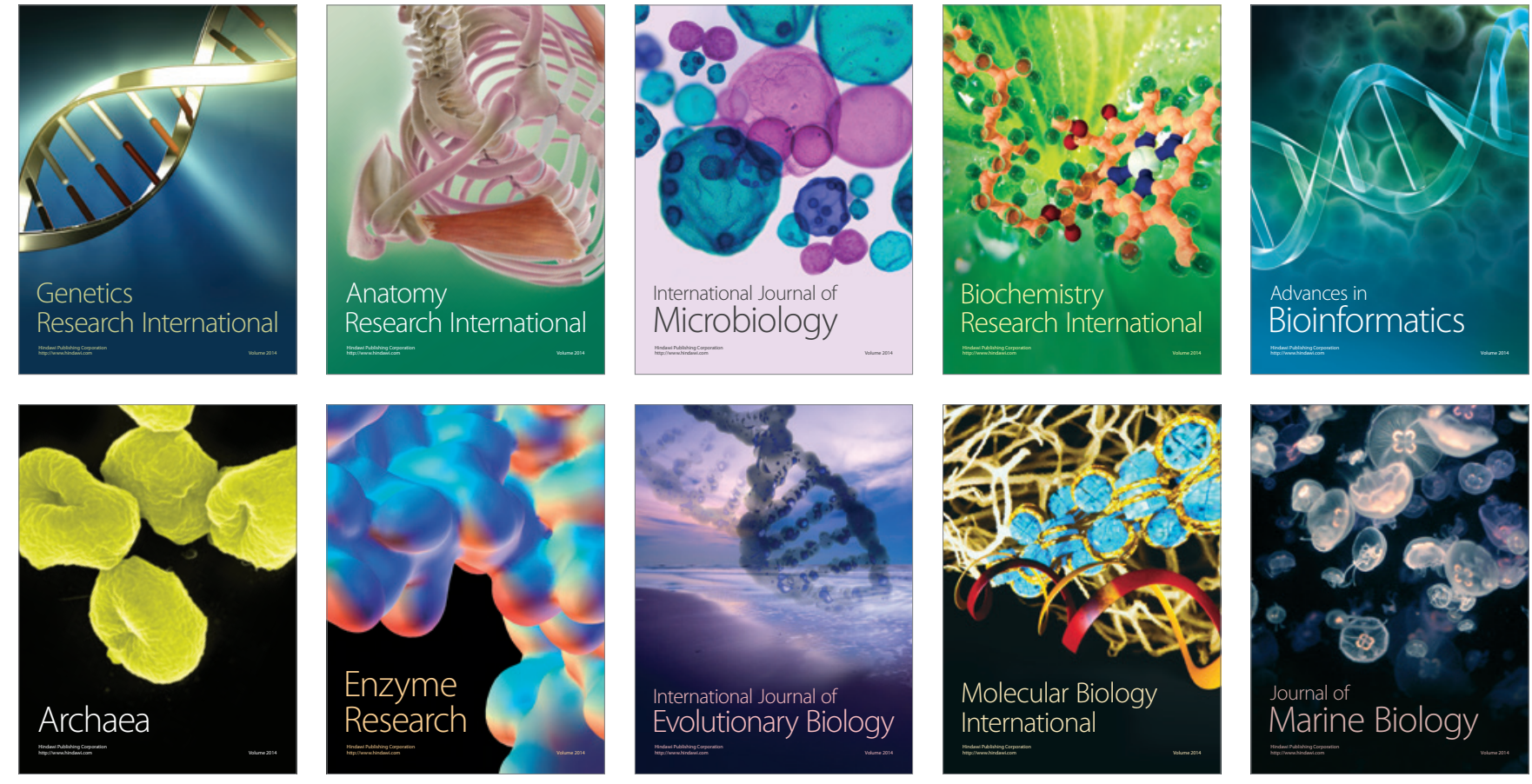